\title{
Remoción del lubricante adicionado al hierro pulvimetalúrgico, con plasma de la descarga luminiscente anormal
}

\author{
Removal of lubricant added to iron powder metallurgy, with \\ abnormal plasma discharge glow
}

Remoção do lubrificante adicionado ao ferro em pó, com plasma da descarga luminescente anormal

Fecha de recepción: 08 de septiembre de 2015

Pedro Vicente Rodríguez-Fonseca*

Fecha de aprobación: 18 de diciembre de 2015 Armando Sarmiento-Santos**

Carlos Mauricio Moreno-Téllez***

\section{Resumen}

Se emplea el plasma de la descarga luminiscente anormal DLA en la remoción del lubricante de compactos de hierro pulvimetalúrgico en atmósfera de hidrógeno, con el fin de establecer las condiciones óptimas del ciclo térmico en la configuración catódica para mantener las propiedades ofrecidas del hierro. Para la preparación de las mezclas se utiliza un mezclador rotatorio. El material se conforma con presión uniaxial en matriz cilíndrica, calculando su densidad y porosidad. Con TGA y DSC se estudian la descomposición térmica y la eliminación del lubricante; luego, se realizan los ciclos de remoción en la DLA. Con TGA se monitorea el estado de remoción del lubricante de cada ciclo en la DLA. Se caracterizan los compactos en verde y los del proceso de remoción con microscopia óptica y SEM.

Se halló una adecuada remoción en la configuración catódica a $400{ }^{\circ} \mathrm{C}$ durante 10 minutos, removiéndose la totalidad de lubricante. El ciclo propuesto conviene en aplicaciones industriales por las altas tasas de calentamiento alcanzadas con reducción del consumo de energía, así como de la necesaria en mantener la descarga, referidas a los hornos resistivos utilizados.

Palabras clave: Hierro pulvimetalúrgico, Pulvimetalurgia, DLA, Plasma de la DLA.

* M.Sc. Universidad Pedagógica y Tecnológica de Colombia (Tunja-Boyacá, Colombia). pedro.rodriguez@uptc.edu.co.

** Ph. D. Universidad Pedagógica y Tecnológica de Colombia (Tunja-Boyacá, Colombia). asarmiento.santos@uptc.edu.co.

*** Ph. D. Universidad Pedagógica y Tecnológica de Colombia, (Tunja-Boyacá, Colombia). carlosmauricio.moreno@uptc.edu.co. 


\section{Abstract}

Abnormal glow discharge plasma is used in removing lubricant from iron powder metallurgical compact in a hydrogen atmosphere, in order to set the best conditions of thermal cycle in the cathodic configuration, to keep the iron characteristics offered. A rotary mixer for preparing the mixtures was used. The material was conformed with uniaxial pressure and calculating its density and porosity. With TGA and DSC, the thermal decomposition and the elimination of the lubricant is studied. The removal cycles are performed on the abnormal glow discharge plasma. With TGA the lubricant removal is monitored. Green compacts and the removal process one's are characterized using optical and SEM microscopy.

Adequate removal in the cathode configuration was achieved at $400{ }^{\circ} \mathrm{C}$ for 10 minutes. The proposed cycle should be considered for industrial applications due to high heating rates reached with reduced power consumption, and the energy required to maintain the discharge, related to the resistance furnaces used.

Keywords: Iron powder metallurgy, Powder metallurgy, DLA, Plasma discharge glow.

\section{Resumo}

Emprega-se o plasma da descarga luminescente anormal DLA na remoção do lubrificante de compactos de ferro em pó em atmosfera de hidrogênio, com o intuito de estabelecer as ótimas condições do ciclo térmico na configuração catódica para manter as propriedades oferecidas do ferro. Para a preparação das misturas se utiliza um misturador rotatório. O material se conforma com pressão uniaxial em matriz cilíndrica, calculando sua densidade e porosidade. Com TGA e DSC se estudam a decomposição térmica e a eliminação do lubrificante; depois, se realizam os ciclos de remoção na DLA. Com TGA se monitora o estado de remoção do lubrificante de cada ciclo na DLA. Caracterizam-se os compactos em verde e os do processo de remoção com microscopia óptica e SEM.

Achou-se uma adequada remoção na configuração catódica a $400{ }^{\circ} \mathrm{C}$ durante 10 minutos, removendose a totalidade de lubrificante. O ciclo proposto é conveniente em aplicações industriais pelas altas taxas de aquecimento alcançadas com redução do consumo de energia, assim como da energia necessária para manter a descarga, referidas aos fornos resistivos utilizados.

Palavras chave: Ferro em pó, Metalurgia do pó, DLA, Plasma da DLA.

Cómo citar este artículo:

[1] P. V. Rodríguez-Fonseca, A. Sarmiento-Santos \& C. M. Moreno-Téllez, "Remoción del lubricante adicionado al hierro pulvimetalúrgico, con plasma de la descarga luminiscente anormal", Fac. Ing., vol. 25 (41), pp. 63-73, ene.-abr. 2016. 


\section{INTRODUCCIÓN}

Los lubricantes de prensado son compuestos que se emplean en el proceso pulvimetalúrgico; su función básica es reducir la fricción entre partículas durante la compactación y la del compacto con las paredes de la matriz y con las herramientas de compactación durante el proceso de prensado y extracción de la pieza [1]. La adición de estos compuestos a los polvos metálicos es necesaria para disminuir el costo de producción, pero tiene numerosas implicaciones sobre las diferentes propiedades de las piezas pulvimetalúrgicas, ya que la presencia de este compuesto en el material por compactar actúa modificando la relación entre la densidad y la presión de compactación (compresibilidad), los gradientes de densidad en el compacto, la transferencia de carga axial y radial, la fuerza de extracción y el desgaste de los elementos de matricería, así como las propiedades finales del compacto sinterizado [2].

Los estearatos de zinc y de litio fueron los primeros lubricantes empleados en la industria pulvimetalúrgica, y, de acuerdo con Lawrence [3], se consideran los mejores para la compactación (prensado y expulsión), dada su gran capacidad lubricadora. También es crucial que cualquier lubricante en el compactado (estearatos que contienen carbono, ceras, zinc, estearatos de litio, etc.) se queme completamente, eliminándolo en un tratamiento de remoción, ya que puede causar carburación durante la sinterización, comprometiendo con ello la resistencia a la corrosión y la maquinabilidad. En general, los lubricantes que contienen un componente metálico tienen que ser tratados con una temperatura más alta que los que son puramente orgánicos.

La buena compresibilidad de los polvos de hierro Ancorsteel 1000 posibilita que con una única compactación y sinterización se obtengan las mayores densidades, lo que permite su adecuado empleo como componente pulvimetalúrgico base para fabricación de piezas o de otros polvos atomizados. El hierro Ancorsteel 1000 suministra, además, excelentes propiedades físicas y mecánicas que se pueden conseguir con pequeñas adiciones de grafito, cobre o níquel; igualmente, garantiza bajos niveles de inclusiones, lo que permite un buen desempeño en aplicaciones de forja [4]; además, su alta compresibilidad daña menos los útiles de compactación. Las características de este polvo de hierro lo hacen idóneo para partes estructurales de alta densidad y como material base para aplicaciones magnéticas, y apto para piezas que requieran una densidad media-alta, con propiedades ligeramente magnéticas; ejemplos de aplicación: pedales y poleas [5-6].

En pulvimetalurgia, una incorrecta eliminación del lubricante puede modificar y reducir, específicamente, las propiedades físicas, químicas y mecánicas de la mezcla metálica, debido a la interacción química que puede presentarse entre los componentes de esta y el lubricante residual. Por tal motivo, el estudio de las condiciones en las que se realiza la eliminación del lubricante es sumamente importante para controlar el proceso. Dada la necesidad de incorporar y, posteriormente, eliminar el lubricante en las mezclas metálicas, hay numerosos estudios sobre los lubricantes; unos con la finalidad de estudiar el modo como afectan las propiedades de la mezcla, tales como segregación, compresibilidad, fluencia y densidad aparente, y otros evaluando los residuos que dejan después de realizar el proceso de presinterización [7].

Ward es uno de los iniciadores en el empleo de técnicas termogravimétricas para el estudio de la remoción del lubricante; específicamente, propone un mecanismo de descomposición del lubricante e identifica el residuo [7-8]. La misma metodología la emplean para concluir que la atmósfera de hidrógeno, bajas velocidades de calentamiento y altas temperaturas favorecen la eliminación de lubricante [9].

La aplicación de la descarga luminiscente anormal al proceso de sinterización es una técnica reciente que representa disminución en el tiempo requerido para el proceso de sinterización de muestras, tanto metálicas como cerámicas, y un menor consumo de energía, debido a que el calentamiento se realiza en forma directa por el bombardeo de los iones y átomos neutros sobre la superficie del cátodo donde generalmente se localiza la muestra [10]. Además de los métodos convencionales de sinterización de los aceros, la sinterización por plasma ha demostrado ser un proceso que puede ser aplicado con éxito [11].

En diversas investigaciones se han propuesto variadas formas de conseguir una remoción eficiente, e incluso se ha propuesto un modelo matemático [12] para establecer las condiciones adecuadas; sin embargo, no hay un estudio concreto respecto del estearato de zinc aplicando la descarga luminiscente anormal para la remoción, aprovechando sus potencialidades 
con características importantes como la uniformidad y reproducibilidad, por lo que es utilizada para tratar superficies y en sinterización.

Este trabajo busca determinar las condiciones de ciclo térmico óptimas para la remoción del lubricante estearato de zinc del hierro pulvimetalúrgico Ancorsteel 1000 empleando el plasma de la descarga luminiscente anormal en DC con presencia de una atmósfera reductora de hidrógeno.

\section{Procedimiento experimental}

Los precursores son polvos atomizados de hierro Ancorsteel 1000 de Hoeganaes y estearato de zinc de Struktol como lubricante; este último en proporción de $0,5 \%$ en peso. Se utilizó un mezclador rotatorio para la preparación de las mezclas a 34 r.p.m. durante 20 minutos. El material se conforma en una compactadora hidráulica con presión de compactación uniaxial de $700 \mathrm{MPa}$ en matriz cilíndrica. Se caracterizan los compactos en verde por su densidad calculada con su masa y dimensiones. Igualmente, se estiman su compacidad, porosidad y compresibilidad. $\mathrm{Se}$ inspeccionan los precursores para determinar su morfología y los compactos en verde para evaluar su porosidad con microscopia óptica y electrónica de barrido SEM.

Se emplearon análisis termogravimétricos (TGA) y de calorimetría diferencial de barrido (DSC) del estearato de zinc de Struktol, con el fin de establecer las probables condiciones de los ciclos térmicos que permitan la eliminación más completa del lubricante. Igualmente, se empleó TGA con un par de los compactos en verde para indagar sobre su pérdida de masa, y TGA de los compactos ya tratados para verificar la presencia de lubricante.

Los compactos en verde se sometieron a la etapa de remoción del lubricante en un reactor de plasma de DLA en la configuración catódica, empleando atmósfera de hidrógeno grado 5,0 con flujo de 200 $\mathrm{mL} / \mathrm{min}$. La presión se fijó en $2 \operatorname{Torr}(266,644 \mathrm{kPa})$. La velocidad de calentamiento fue de $100^{\circ} \mathrm{C}$ por minuto. Los parámetros evaluados fueron los ciclos térmicos durante el proceso de remoción del lubricante a temperaturas de 300,400 y $500{ }^{\circ} \mathrm{C}$ y tiempos de permanencia de 10,20 y 30 minutos una vez alcanzadas las temperaturas. Se enfriaron los compactos en el interior del horno hasta la temperatura ambiente, para su protección, evitando la oxidación del hierro.

Los compactos sometidos a la remoción son evaluados por su pérdida de masa y caracterizados a través de microscopía electrónica de barrido SEM con el fin de revisar su porosidad y establecer la posible presencia de residuos del lubricante sobre los compactos.

\section{Resultados}

Alrededor de la caracterización de los precursores, se cuenta con la información suministrada por los fabricantes. En la Tabla I se anota la composición química de los polvos de hierro Ancorsteel 1000, como porcentaje en peso; su densidad aparente es de $2,94 \mathrm{~g} / \mathrm{cm}^{3}$ y su velocidad de flujo de $26 \mathrm{~s} / 50 \mathrm{~g}$.

\section{TABLA I}

CoMposición del HIERRo ANCORSTEEl 1000 (PORCENTAJE EN PESO) (w/o) [4]

\begin{tabular}{|c|c|c|c|c|c|c|c|c|c|}
\hline $\mathrm{C}$ & $\mathrm{O}$ & $\mathrm{N}$ & $\mathrm{S}$ & $\mathrm{P}$ & $\mathrm{Si}$ & $\mathrm{Mn}$ & $\mathrm{Cr}$ & $\mathrm{Cu}$ & $\mathrm{Ni}$ \\
\hline$<0.01$ & 0.14 & 0.002 & 0.018 & 0.009 & $<0.01$ & 0.20 & 0.07 & 0.10 & 0.08 \\
\hline
\end{tabular}

En la Tabla II se indica la distribución granulométrica de los polvos de hierro Ancorsteel 1000 por tamaños en porcentaje en peso. 


\section{TABLA II}

DistribuCión GRANUlOMÉTRICA DEL HIERRo ANCORSTEEL 1000 [4]

Sieve Distribution (w/0)
\begin{tabular}{|l|c|c|c|c|}
\hline Micrometers & +250 & $-250 /+150$ & $-150 /+45$ & -45 \\
\hline U.S. Standard Mesh & $(+60)$ & $(-60 /+100)$ & $(-100 /+325)$ & $(-325)$ \\
\hline & Trace & 10 & 68 & 22 \\
\hline
\end{tabular}

La Fig. 1 muestra el efecto de la presión de compactación sobre la densidad en verde y el efecto de esta sobre la resistencia en verde del hierro Ancorsteel 1000.
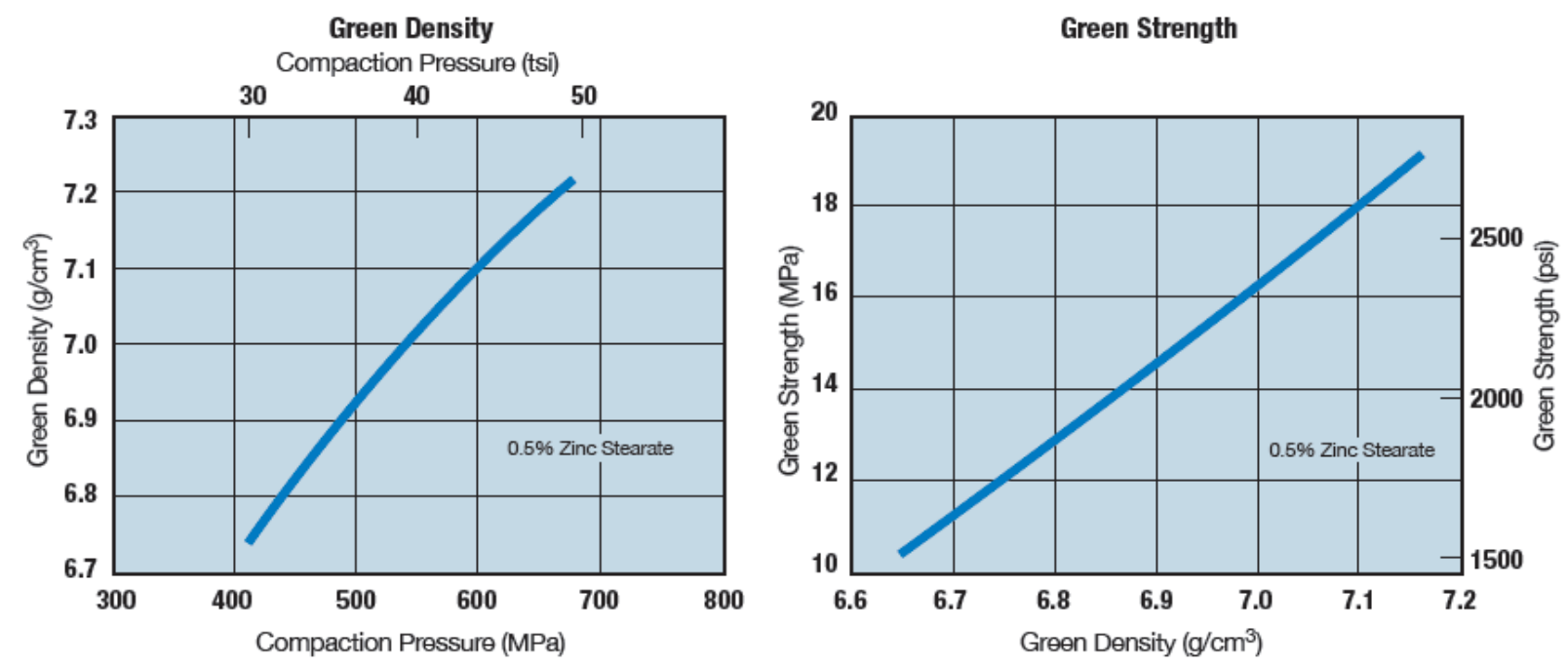

Fig. 1. Efecto de la presión de compactación sobre la densidad en verde y de la densidad en verde sobre la resistencia en verde del hierro Ancorsteel 1000 [4].

Los estearatos son añadidos a los polvos metálicos como una pequeña forma esférica (normalmente atomizados). Las propiedades del lubricante estearato de zinc común están dadas en la Tabla III.

\section{TABLA III}

Propiedades del lubricante común de estearato de ZinC en P/M [13]

\begin{tabular}{|l|c|c|c|c|c|}
\hline $\begin{array}{c}\text { Nombre } \\
\text { común }\end{array}$ & Óxido & $\begin{array}{c}\text { Porcentaje de } \\
\text { óxido }\end{array}$ & $\begin{array}{c}\text { Temperatura de } \\
\text { ablandamiento, }{ }^{\circ} \mathbf{C}\end{array}$ & $\begin{array}{c}\text { Temperatura de } \\
\text { fusión, }{ }^{\circ} \mathbf{C}\end{array}$ & $\begin{array}{c}\text { Densidad, } \\
\mathbf{g} / \mathbf{c m}^{\mathbf{3}}\end{array}$ \\
\hline $\begin{array}{l}\text { Estearato de } \\
\text { zinc }\end{array}$ & $\mathrm{ZnO}$ & 14 & 100 a 120 & 130 & 1.09 \\
\hline
\end{tabular}

Propiedades típicas del estearato de zinc en polvo de Struktol se listan en la ficha técnica del fabricante, observando que su punto de fusión se encuentra en el rango de 117 a $123{ }^{\circ} \mathrm{C}$, su contenido de humedad máximo es del 1,0\% y el tamaño de las partículas se caracteriza porque atraviesan la malla 325 en un $99 \%$ [14].

Las partículas de los polvos fueron inspeccionadas empleando microscopía electrónica de barrido SEM para revisar su tamaño y morfología. Se observa una 
morfología irregular de las partículas tanto del hierro Ancorsteel 1000 como del estearato de zinc, las cuales son típicas de sus procesos de fabricación, con una diferencia en sus bordes, redondeados en el hierro atomizado y angulares para el estearato de zinc (Fotos 1 y 2 ).

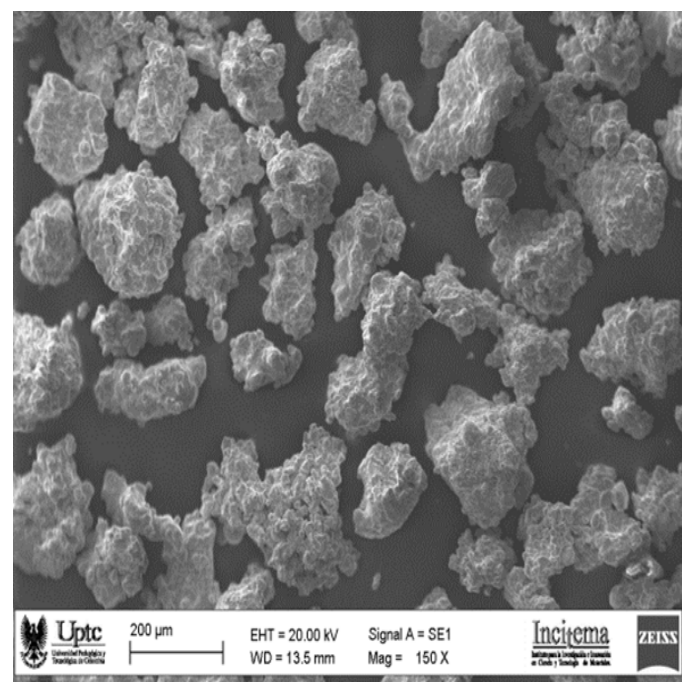

Foto 1. SEM de los polvos de hierro Ancorsteel 1000.

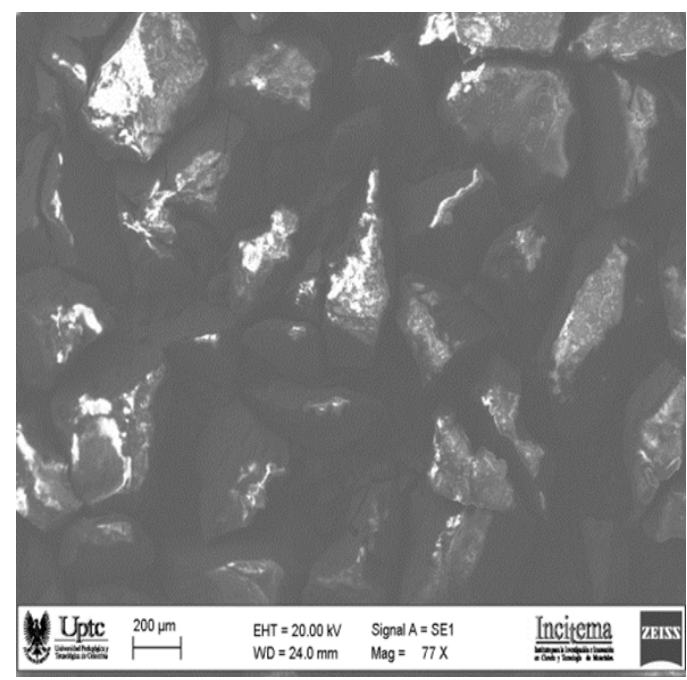

Foto 2. SEM de los polvos del estearato de zinc de Struktol.
Las diferentes mediciones y pesajes de los compactos se realizaron por triplicado y se promediaron. Se encontraron densidades que están entre 6,2139 y $7,0392 \mathrm{~g} / \mathrm{cm}^{3}$, las cuales representan un rango del 78,99 a $89,48 \%$ de la densidad real del hierro y un rango del 91,38 a más del $100,00 \%$ de la densidad en verde alcanzable para el hierro Ancorsteel 1000, incluyendo $0,5 \%$ en peso del lubricante estearato de zinc. Los rangos especialmente bajos pueden obedecer a la necesidad que se tuvo de pulir los bordes de algunos de los compactos que presentaron rebabas principalmente por una de sus caras.

Los valores de compacidad oscilaron entre 83,9771 y $87,5349 \%$, que se encuentran como normales, tomando como densidad real $7,87 \mathrm{~g} / \mathrm{cm}^{3}$, densidad informada del hierro Ancorsteel 1000 [4]. Asimismo, la porosidad en verde está en el rango de 12,4651 a $16,0229 \%$, y su compresibilidad, entre 55,5152 y $57,3233 \%$.

Utilizando la microscopía óptica entre 100 y 500 aumentos con los compactos en verde y los sometidos a remoción, se observa una porosidad irregular conforme a los contornos de los polvos, porosidad primaria abierta e interconectada, de distribución uniforme (Foto 3).

Previo a la realización de los diferentes tratamientos para la remoción del lubricante, se analizó el resultado del TGA y DSC para el estearato de zinc, con el fin de encontrar las mejores condiciones de ciclos térmicos para el proceso (Fig. 2). Con base en el análisis del resultado obtenido, se encontró que a $300^{\circ} \mathrm{C}$ no era práctico efectuar la remoción, lo que se comprobó con un par de ensayos a esta temperatura tomando los diferentes tiempos de sostenimiento $(10,20 \mathrm{y}$ 30 minutos) y observando posteriormente, por datos arrojados a través de TGA, que aún se mantenía una cantidad importante del lubricante; pero llegando a los $400{ }^{\circ} \mathrm{C}$ se encontró que ya se ha removido más del $50 \%$ del lubricante; temperatura a la que hacen referencia algunos autores como la temperatura $\mathrm{T}_{50}$, la cual es importante superar (Fig. 3) [12]. 


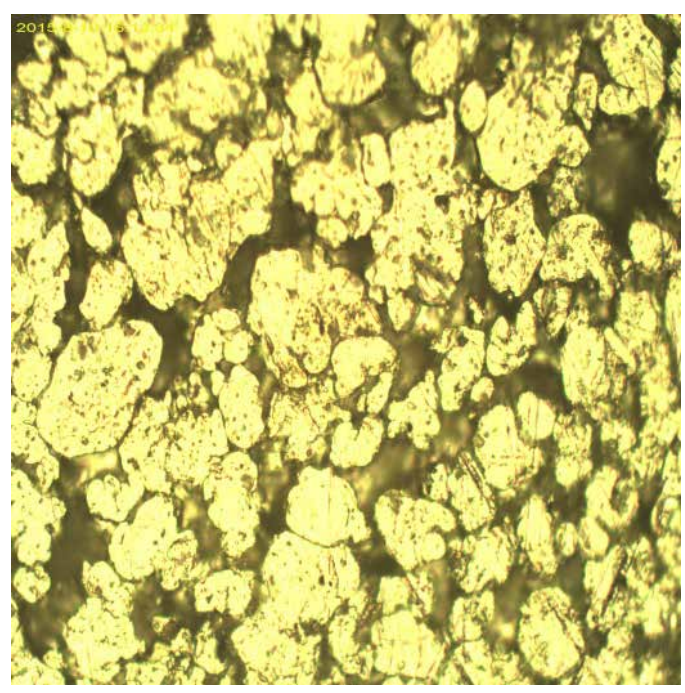

(a)

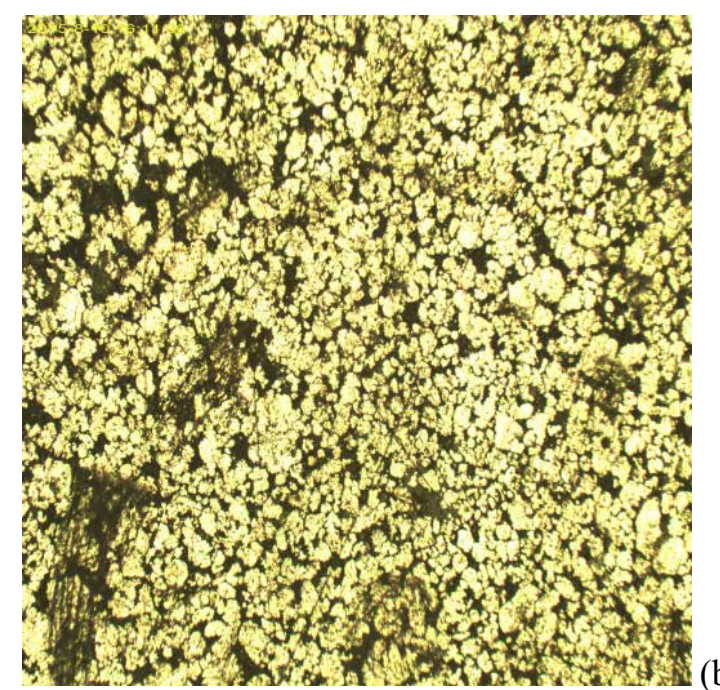

Foto 3. Micrografía a 500X (a) y a 100X (b) de compacto en verde.

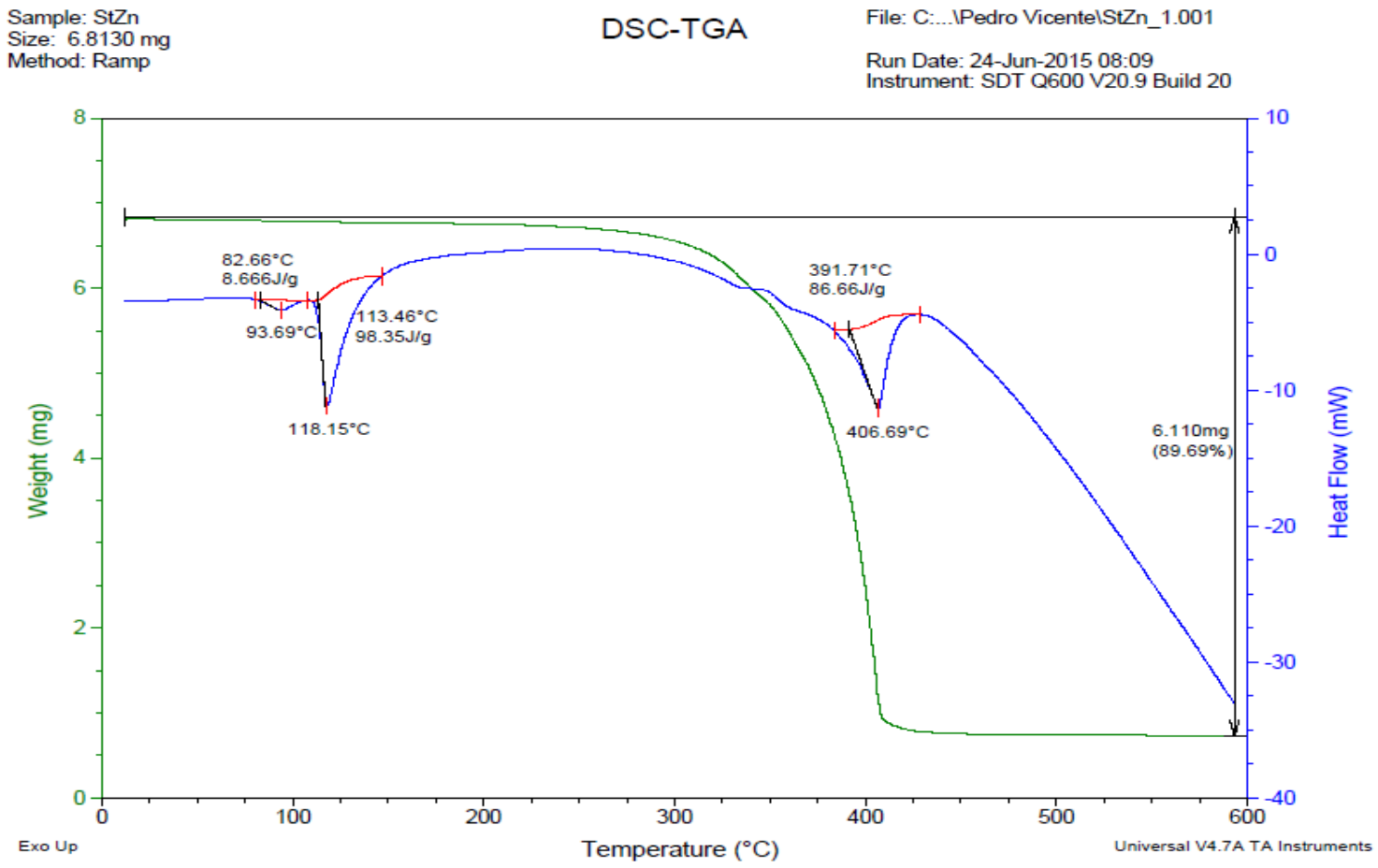

Fig. 2. TGA y DSC del estearato de zinc. 


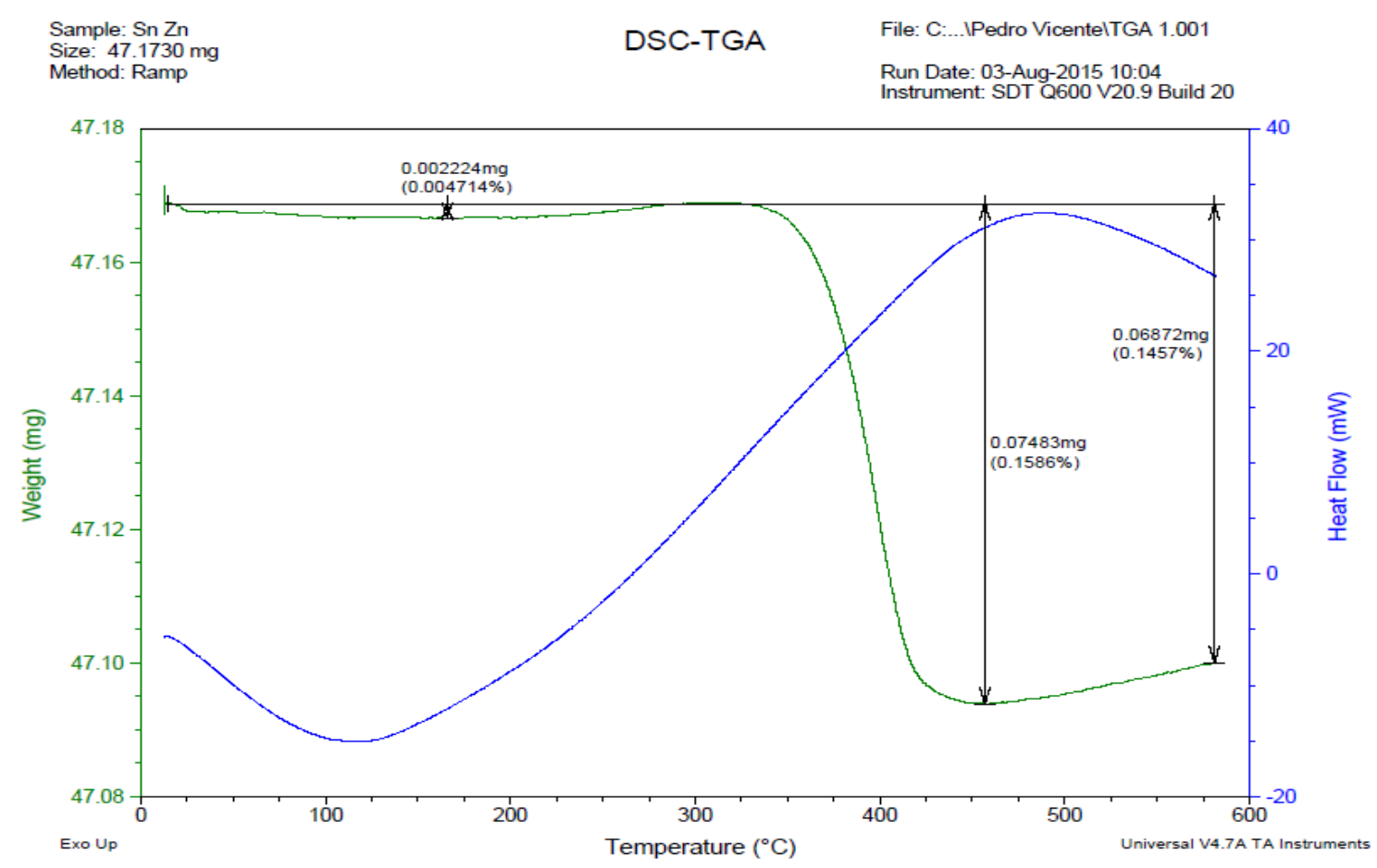

Fig. 3. TGA y DSC del compacto verde con tasa de calentamiento de $40^{\circ} \mathrm{C} / \mathrm{min}$.

La determinación de la temperatura $\mathrm{T}_{50}$ es atribuida a la eliminación de la mitad del lubricante [12-15,16], y es a partir de la cual se acelera el proceso de remoción de este. De acuerdo con el resultado del TGA de la Fig. 2, esta temperatura para el estearato de zinc está alrededor de los $400{ }^{\circ} \mathrm{C}$, bastando, por tanto, con sostener esa misma temperatura un tiempo adecuado para su remoción máxima.

Los porcentajes de remoción se hallan en valores del 0,3513 al $0,4407 \%$ en promedio. Se deduce que no se encuentra un valor promedio del $0,5 \%$ como era de esperarse a pesar de tener valores puntuales hasta de $0,6896 \%$ con un tratamiento a $400{ }^{\circ} \mathrm{C}$ y 10 minutos de permanencia, lo que se explica como una posible segregación de la distribución de las partículas en la mezcla, en particular del lubricante que tiende a aglomerarse, lo que pudo llevar a mayor presencia de lubricante en algunos de los compactos y a menor en otros, que presentaron valor puntual hasta de $0,2523 \%$ para un tratamiento de $500{ }^{\circ} \mathrm{C}$ y 30 minutos de permanencia.

Teniendo en cuenta que algunos autores plantean que al remover el $97 \%$ del lubricante se considera que ha sido totalmente eliminado, tendríamos, como consideración práctica [12-15], que una pérdida de masa del $0,485 \%$ sería absolutamente satisfactoria para un contenido teórico de $0,5 \%$ del lubricante, lo cual se consiguió en algunos casos puntuales, especialmente en los tratamientos efectuados a $400{ }^{\circ} \mathrm{C}$ de 10 y 30 minutos de permanencia. No obstante, aplicando la estadística a todos los tratamientos se observa que la mejor remoción se obtuvo con $400^{\circ} \mathrm{C}$ y 10 minutos de permanencia, seguida del tratamiento a $400{ }^{\circ} \mathrm{C}$ y 30 minutos de duración.

La mejor remoción a $500{ }^{\circ} \mathrm{C}$ se logró con una permanencia de 20 minutos, pero las remociones más bajas fueron a la misma temperatura, con $30 \mathrm{y}$ 10 minutos de permanencia; esta última presenta la menor remoción del lubricante.

Caso particular son los tratamientos realizados a 500 ${ }^{\circ} \mathrm{C}$, en los que, a pesar de tener mayor temperatura, se consiguieron remociones en apariencia inferiores, lo que lleva a pensar en la posibilidad de una ganancia de masa debida a factores como el sputtering y el intercambio atómico del material del ánodo, del cátodo y del compacto. Incluso, en el momento de superar los $425^{\circ} \mathrm{C}$ [17], cuando ya se ha presentado la evaporación del lubricante, como se observó en el análisis TGA del estearato de zinc, se presentan reacciones de sus vapores con la atmósfera, cuyos 
productos se empiezan a depositar en la superficie del compacto en forma de óxido de zinc [7] o por óxidoreducción del hierro, a partir del oxígeno que escapa del estearato y del hidrógeno de la atmósfera, o como hidrocarburo en evaporación.

Se observó en el desarrollo de los tratamientos que, regularmente, a partir de una temperatura sobre los $150^{\circ} \mathrm{C}$ se iniciaba un comportamiento de arco que se mantenía hasta alrededor de $\operatorname{los} 300^{\circ} \mathrm{C}$, probablemente como manifestación de las interacciones por pulverizaciones catódicas (sputtering) al comenzar la salida del lubricante estearato de zinc fluido a la superficie del compacto expuesta a la atmósfera. En uno de los ensayos se mantuvo la temperatura en estos rangos y se encontró que se estabilizó la descarga en un tiempo inferior a los 5 minutos.

Es probable que en el lapso de temperatura mencionado se depositen unas coloraciones superficiales en buena parte de los compactos, las cuales se presentan en una capa muy fina (azulosa) que se puede observar en las micrografías de compactos con el tratamiento de remoción (Fotos 4 y 5). La capa formada sobre la mayoría de los compactos tratados se encuentra en extensiones diversas, quizá de acuerdo con la proporción del lubricante eliminado.

Se emplea SEM con una sonda EDS para determinar la composición de las películas superficiales formadas sobre la mayoría de los compactos con tratamiento de remoción; uno de los espectros de área se muestra en la Fig. 4.

Tanto los espectros de área como los puntuales realizados indican la presencia de $\mathrm{ZnO}$, residuo de la remoción del estearato de zinc, en proporciones que son aproximadas a las del contenido de este compuesto en el lubricante. En la Foto 6 se observa el área analizada.

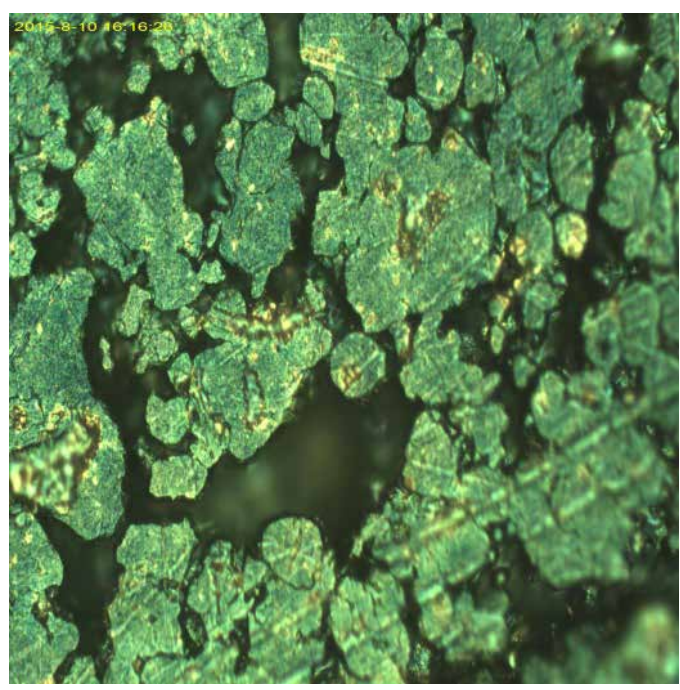

Foto 4. Micrografía a 500X de compacto con remoción.

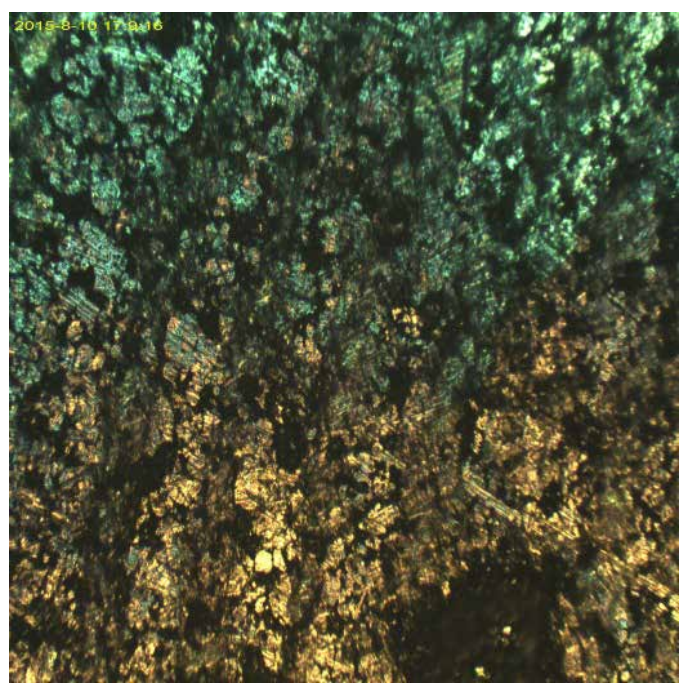

Foto 5. Micrografía a 100X de compacto con remoción. 


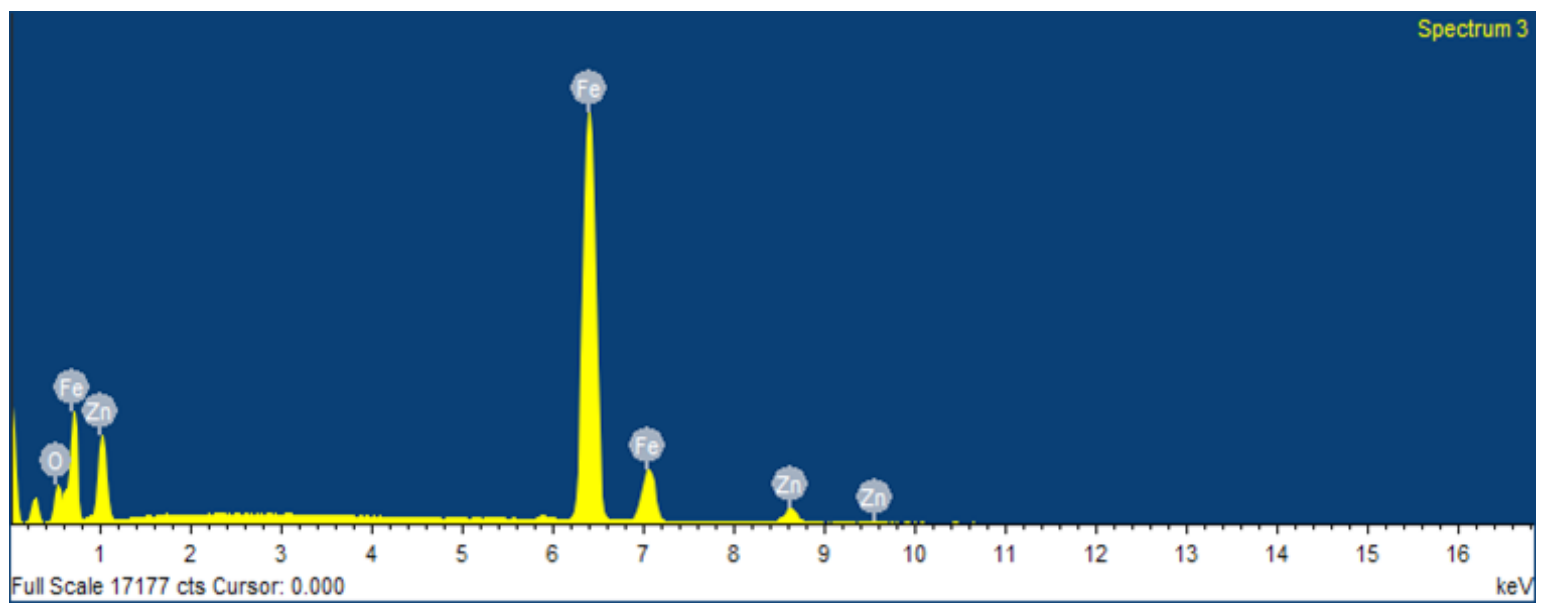

Fig. 4. Espectro SEM del análisis elemental de área en compacto con remoción tratado a $400{ }^{\circ} \mathrm{C}$ y 10 minutos de permanencia.

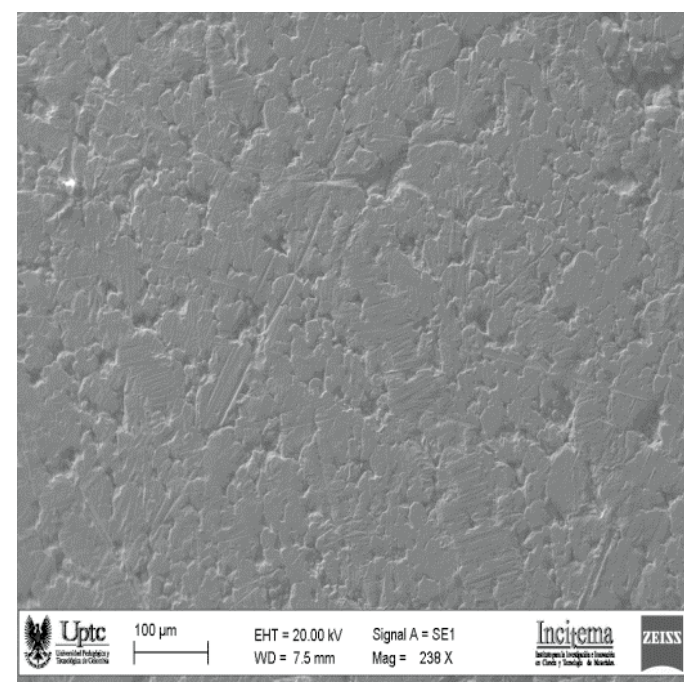

Foto 6. Micrografía SEM de compacto con remoción (Tratamiento a $500{ }^{\circ} \mathrm{C}$ con 20 minutos de sostenimiento).

\section{Conclusiones}

Para facilitar una mejor eliminación del lubricante resulta importante conseguir una distribución homogénea de él en la masa del compacto y una porosidad adecuada, mejorando la presentación de los compactos sin crear interacciones indeseables e inesperadas en el interior del reactor de sinterización, independiente de la clase de reactor empleado para el proceso.

Es importante prestar atención a la granulometría y distribución de los polvos empleados como precursores, y, de ser requerido, efectuar una clasificación previa de estos a fin de lograr una porosidad y densidades apropiadas, y una correcta distribución de los materiales en la matriz en la que se conformen las piezas.

Los resultados ponen en evidencia el alto potencial de empleabilidad de la tecnología del plasma para la conveniente eliminación del lubricante a través de un ciclo térmico pertinente en la configuración catódica tradicional.

El ciclo térmico que permite la mayor eliminación del lubricante estearato de zinc de los compactos de hierro pulvimetalúrgico para la sinterización con plasma de descarga luminiscente es el calentamiento a una velocidad elevada de $100{ }^{\circ} \mathrm{C} /$ minuto hasta la temperatura de $400{ }^{\circ} \mathrm{C}$ y su permanencia durante 10 minutos, empleando la configuración catódica de la descarga anormal.

A nivel industrial conviene hacer uso de ventajas como la de un rápido proceso en los reactores de plasma con una alta eficiencia de la energía para los precursores evaluados con sus características de uniformidad y reproducibilidad, generando un bajo impacto ambiental.

En casi todos los casos se encontraron sobre los compactos tratados residuos del lubricante, en ocasiones en forma de "hollín" y generalmente como una película superficial no siempre uniforme sobre el compacto, de color azulado, que corresponde a óxido de zinc. El estearato de zinc deja residuos en cualquiera 
de las condiciones del tratamiento de acuerdo con su composición estequiométrica.

Queda planteada una guía para el tratamiento de remoción más eficiente del estearato de zinc utilizado como lubricante en el hierro pulvimetalúrgico, que mejora la productividad y economía del proceso sin que se presente problema por daño de los compactos.

\section{REFERENCIAS}

[1] S. Amstrong, M. D. Aesoph and A. L. Gurson, "The Effects of Lubricant Content and Relative Powder Density on the Elastic, Yield and Failure Behavior of a Compacted Metal Powder", Advances in Powder Metall, Metal Powders Industries Federation, vol. 3, pp. 31, 1995.

[2] M. Ward, "Influence of Lubricants on Dimensional Changes and Mechanical Properties of Sintered Ferrous Compacts", Powder Metall. IoM Communications, no. 4, pp. 193, 1979.

[3] A. I. Lawrence et al., "A performance Comparison of Current $\mathrm{P} / \mathrm{M}$ Lubricants and Routes to Improvement", Advances in Powder Metall, Compiled by C. Lall and A.J. Neupaver, Metal Powders Industries Federation, vol. 3, pp. 103, 1997.

[4] Hoeganaes Corporation, Data Sheet: Ancorsteel 1000, pp. 1-5, 0613-AS1000-D-2.

[5] Hogänäs AB., Materials and Powders Properties, PoP Application Center of Hogänäs, Handbook No. 1, Chapter 1, 2007.

[6] Hogänäs AB., Compacting of Metal Powders, PM School, Handbook No. 2, Chapter 4 and Chapter 6, 2007.

[7] A. Bolarín et al., Análisis Termogravimétrico del Proceso de Eliminación de Lubricante en Compactos $P / M$ ', Centro de Investigaciones de Materiales y Metalurgia - Universidad Autónoma del Estado de Hidalgo (UAEH), Universidad Autónoma de Coahuila, México, 2015.
[8] M. Ward, "Decomposition Characteristics of some lubricants in iron $\mathrm{P} / \mathrm{M}$ compacts as a free compounds", Int. J. Powder Metallurgy and Powder Tech, vol. 3, no. 3, pp. 197, 1977.

[9] Kuen-Shyang and Li Kuang-Hung, "Lubricant Removal in Metal Powder Compacts", The International Journal of Power Metallurgy, Vol. 28, no. 4, 1992.

[10] A. Sarmiento S. y J. L. R. Muzart, "Tratamiento de Acero 316L en Descarga Luminiscente Anormal", Revista Colombiana de Física, vol. 40, no. 2, pp. 427-429, julio 2008.

[11] J. L. R. Muzart et al. Advances in Powder Metallurgy \& Particulate Materials, part. 3, pp. 77-84, 1997.

[12] D. Saha and D. Apelian, "Control Strategy for De-lubrication of $\mathrm{P} / \mathrm{M}$ Compacts", The International Journal of Powder Metallurgy, Vol. 38, no. 3, pp. 5-7, 13-15, 17-21, 2002.

[13] G. Randall M., Powder Metallurgy Science, 2th ed., Princeton, New Jersey, U.S.A., Metal Powder Industries Federation, 1994.

[14] Struktol Company of America, Technical Data.

[15] Arnaud Gateaud, Physical and Chemical Mechanisms of Lubricant Removal During Stage I of the Sintering Process, Directores: Diran Apelian, Richard D. Sisson, Thesis Degree of Master of Science in Materials Science and Engineering, Worcester Polytechnic Institute, 2006.

[16] D. Saha, De-lubrication during Sintering of P/M Compacts: Operative Mechanism and Process Control Strategy, Directores: D. Apelian, Richard D. Sisson, Thesis Degree of Master of Science in Materials Science and Engineering, Worcester Polytechnic Institute, pp. 5, 2001.

[17] E. Hryha et al., "Influence of the Steel Powder TypeandProcessing Parameters on theDebinding of PM Compacts with Gelatin Binder", Journal of Thermal Analysis and Calorimetry, Vol. 118, Issue 2, pp. 695, november 2014. DOI: http:// dx.doi.org/10.1007/s10973-014-3839-7. 